\title{
Correlations of chemokine CXCL16 and TNF- $\alpha$ with coronary atherosclerotic heart disease
}

\author{
JIEYONG XING, YANSHAO LIU and TAO CHEN \\ Department of Cardiovascular Medicine, Jinan Zhangqiu Hospital \\ of Traditional Chinese Medicine, Jinan, Shandong 250200, P.R. China
}

Received July 13, 2017; Accepted October 31, 2017

DOI: $10.3892 /$ etm.2017.5450

\begin{abstract}
This study determined the correlations of CXC ligand 16 (CXCL16) and tumor necrosis factor- $\alpha$ (TNF- $\alpha$ ) levels with coronary atherosclerotic heart disease (CAHD) and screened for new clinical markers for the prognosis and treatment of the disease. Eighty patients with coronary heart disease and 50 healthy subjects were enrolled into a CAHD or healthy control group, respectively. Computed tomography (CT) coronary angiography and Gensini integral were used to classify plaques and evaluate patients with coronary heart disease. The serum levels of CXCL16 and TNF- $\alpha$ of subjects in each group were detected by enzyme-linked immunosorbent assays (ELISA), and the correlation between levels and clinical markers (such as blood pressure, glucose, lipid and heart rate) and the severity of disease were analyzed. Our results showed the serum levels of CXCL16 and TNF- $\alpha$ were significantly higher in the CAHD group than those in the CK group. The serum CXCL16 levels of the CAHD group patients with plaques were distinctly higher than those of the CADH group patients without plaques, but there were no significant difference in serum TNF- $\alpha$ levels between these two groups of patients. The level of CXCL16 had a significantly positive correlation with the severity of disease, but there was no significant correlation between TNF- $\alpha$ level and the severity of disease. Also, there was no significant correlation between the CXCL16 levels and blood pressure, blood glucose, heart rate, total cholesterol, triglyceride or high-density lipoprotein cholesterol, but there was a clear correlation with the low-density lipoprotein cholesterol. Finally no significant correlations were found between TNF- $\alpha$ levels and each of the clinical markers studied. Based on our findings, the levels of CXCL16 and TNF- $\alpha$ in the patients with coronary heart disease were abnormally increased and the level of CXCL16 correlated closely with the severity of disease. These markers seem to be reliable biological markers for prognosis and disease evaluation in coronary heart disease patients.
\end{abstract}

Correspondence to: Dr Tao Chen, Department of Cardiovascular Medicine, Jinan Zhangqiu Hospital of Traditional Chinese Medicine, 1463 Xiushui Street, Zhangqiu, Jinan, Shandong 250200, P.R. China E-mail: chentao753357abc@163.com

Key words: chemokine CXCL16, TNF- $\alpha$, coronary atherosclerotic heart disease, correlation

\section{Introduction}

Coronary atherosclerotic heart disease (CAHD), coronary heart disease for short, is due to coronary artery narrowing or obstruction caused by coronary atherosclerotic lesions and plaque formation in the lumen, that reduce the flow of oxygen rich blood to the heart, resulting in heart ischemia, anoxia or necrosis (1). The pathogenesis of CAHD is excessively complex, but is generally based on lumen stenosis or occlusion caused by atherosclerosis (AS), thus impeding normal blood circulation. Recent studies have shown the inflammatory response plays an important role in the occurrence and development of AS (2,3). Vascular inflammatory responses can change the shape of the vascular wall, initiate thrombogenesis and promote the transformation of AS plaques from a stable to a fragile state, thus inducing embolism and incurring a variety of cardiovascular and cerebrovascular diseases (4).

Cytokines are small-inducible proteins synthesized and secreted by body cells upon stimulation. They are involved in cell growth and differentiation, regulation of inflammation and immune response and other processes; there are many kinds of cytokines including interferon, interleukin, chemokine and tumor necrosis factor (5). Recent studies have indicated that chemokine CXC ligand 16 (CXCL16) participates in the pathologic process of AS through its regulation of the inflammatory response, lipid metabolism and acceleration of AS plaque thrombogenesis vulnerability (6-8). Tumor necrosis factor- $\alpha(\mathrm{TNF}-\alpha)$ is secreted by monocyte-macrophages and can be involved in the immune response and the regulation of inflammatory responses (9).

CXCL16 and vascular inflammation have been shown to play important roles in AS lesions (10), so we speculated that its levels in serum would be closely related to the presence of CAHD. We determined expression levels of chemokine CXCL16 and TNF- $\alpha$ in patients with CAHD and analyzed the correlation between those levels and the severity of disease and clinical markers, so as to provide new ideas for prognosis and treatment of CAHD.

\section{Patients and methods}

Materials

General data. Eighty patients with CAHD diagnosed by the Department of Cardiology in Jinan Zhangqiu Hospital 
of Traditional Chinese Medicine from March 2015 to December 2016 were selected and set as a CAHD group. This included 52 males and 28 females, with an average age of $61.73 \pm 12.54$ years. At the same time, 50 healthy subjects undergoing physical examinations in the outpatient department were enrolled and set as the healthy control group, with 31 males and 19 females and an average age of $60.21 \pm 10.79$ years. The diagnostic criteria for patients with CAHD met those for the diagnosis of ischemic heart disease formulated by the World Health Organization (WHO) in 1979, and were confirmed by computed tomography (CT) coronary angiography. Patients with a coronary artery (or any vascular branch) diameter stenosis degree of $>50 \%$ were regarded as positive. Patients with diabetes or hypercholesterolemia were excluded from the CAHD group, while patients with cardiovascular or endocrine disease were excluded from the healthy control group. All subjects belonged to the Han ethnicity and were unrelated individuals and there were no significant differences in gender ratios or age between the two groups ( $p>0.05)$. The study was approved by the Ethics Committee of Jinan Zhangqiu Hospital of Traditional Chinese Medicine and informed consents were signed by the patients and/or guardians.

Main reagents. Assay kits for serum total cholesterol (TC), triglyceride (TG), ELISA, low-density lipoprotein cholesterol (LDL-C) and high-density lipoprotein cholesterol (HDL-C) were purchased from Beijing Solarbio Science and Technology (Beijing, China). Blood glucose assay kit (Beijing Leadman Biochemical Technology Co., Ltd., Beijing, China).

\section{Methods \\ CT coronary angiography and disease evaluation. Coronary arteriography was performed by the digital subtraction angi- ography method. Subjects with vascular diameter stenosis degrees of $>50 \%$ of a coronary artery or any of its branches were classified as CAHD patients. The number of affected vessels was recorded and the Gensini integral was used to evaluate the severity of the disease. The CAHD group was divided into plaque $(n=56,37$ males and 19 females, with an average age of $63.45 \pm 9.74$ years) and no-plaque group $(\mathrm{n}=24,15$ males and 9 females, with an average age of $60.33 \pm 12.31$ years).}

Specimen collection. Fasting venous blood samples (3-4 ml) were extracted from patients with CAHD on the second day of admission and from healthy individuals on the day of physical examination. The samples were placed into centrifuge tubes, followed by centrifugation at 2,000 rpm at room temperature for $10 \mathrm{~min}$. Subsequently, the upper sera were transferred into fresh centrifuge tubes and stored at $-80^{\circ} \mathrm{C}$. All the samples were treated together by one-time subsequent detection after all the collections were completed.

Detection of serum concentrations of CXCL16 and TNF- $\alpha$. The serum levels of CXCL16 and TNF- $\alpha$ in each group were detected by enzyme-linked immunosorbent assay (ELISA), referring to operation instructions in the serum CXCL16 and serum TNF- $\alpha$ quantitative assay kits by R\&D Systems (Minneapolis, MN, USA).
Detection of clinical markers. The routine clinical markers, such as blood pressure and heart rate were detected using standard hospital instruments. Levels of laboratory biochemical markers, such as seric TC, TG, LDL-C and HDL-C were determined by following the instructions in the assay kits as above-mentioned in the CT coronary angiography and disease evaluation.

Statistical analysis. SPSS 17.0 software (SPSS, Inc., Chicago, IL, USA) was used for statistical analysis. The t-test was adopted to analyze the normal distribution of measurement data. Data that conformed to the normal distribution were represented as mean \pm standard deviation and those that did not conform to the normal distribution were expressed by median (interquartile range) $[\mathrm{M}(\mathrm{Q})]$. The independent sample t-test was used to compare the mean of the two groups and the single factor analysis of variance was adopted to compare the mean among multiple groups. Spearman's rank correlation analysis was used to analyze the correlation between serum levels of CXCL16 and TNF- $\alpha$ and the severity of the disease and specific clinical signs. $\mathrm{P}<0.05$ was considered to indicate a statistically significant difference.

\section{Results}

Comparisons of serum levels of CXCL16 and TNF- $\alpha$ between the CAHD and the healthy control groups. The serum levels of CXCL16 and TNF- $\alpha$ were both significantly higher in the CADH group, when quantified by ELISA (Fig. 1).

Comparisons of serum levels of CXCL16 and TNF- $\alpha$ between the plaque and no-plaque groups within the CAHD group. The patients in the $\mathrm{CADH}$ group were divided into a plaque or no-plaque subgroup according to the results of an ultrasound examination. While the serum CXCL16 levels were significantly higher in the plaque than that in the no-plaque group the levels of TNF- $\alpha$ were not significantly different among the groups (Fig. 2).

Correlation between serum levels of CXCL16 and TNF- $\alpha$ and severity of the disease. The Gensini score was used to classify the coronary lesion in CAHD patients, to assess the severity of CAHD. The correlation between the Gensini score and the serum levels of CXCL16 and TNF- $\alpha$ was analyzed, the results are shown in Fig. 3. The serum CXCL16 level has a significantly positive correlation with the Gensini score $(r=0.5970, p=0.0002)$; and the serum TNF- $\alpha$ level is not significantly correlated with Gensini score $(r=-0.1214$, $\mathrm{p}=0.4872$ ).

Correlation between serum levels of CXCL16 and TNF- $\alpha$ and CAHD clinical variables. The results of correlation analyses between serum levels of CXCL16 and TNF- $\alpha$ and each clinical variable in patients with CAHD are shown in Table I. The serum CXCL16 level is not significantly correlated with systolic pressure, diastolic pressure, fasting blood glucose, heart rate or TC, TG and HDL-C levels, but has a significantly positive correlation with the LDL-C level ( $r=0.597, \mathrm{p}=0.027)$. There were no significant correlations between serum TNF- $\alpha$ levels and each clinical variable. 
A

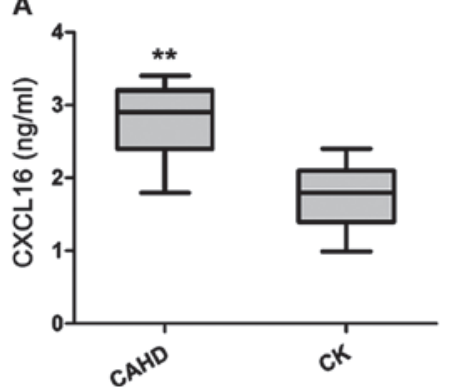

B

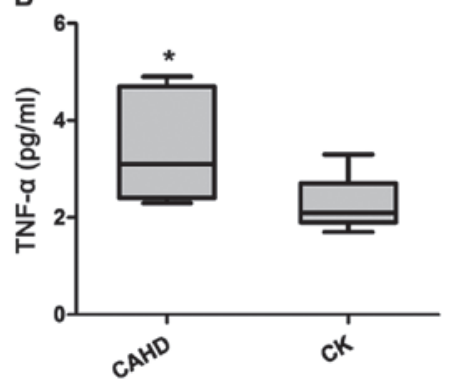

Figure 1. Serum levels of CXCL16 and TNF- $\alpha$ in the CAHD group and CK group. (A) Serum CXCL16 concentration. (B) Serum TNF- $\alpha$ concentration. ${ }^{*}$ p $<0.05$ vs. healthy control; ${ }^{* *}$ p $<0.01$ vs. healthy control. CXCL16, CXC ligand 16; TNF- $\alpha$, tumor necrosis factor- $\alpha$; CAHD, coronary atherosclerotic heart disease.

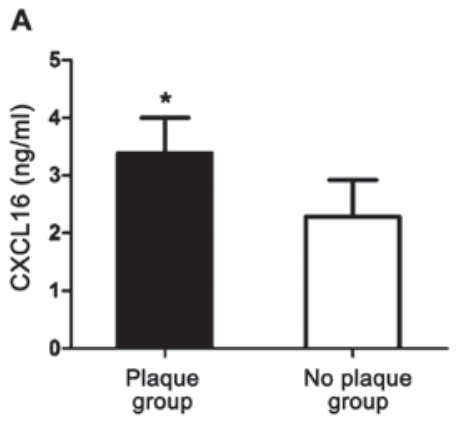

B

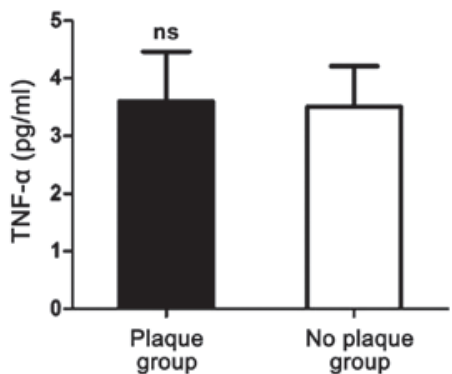

Figure 2. Serum levels of $\mathrm{CXCML16}$ and $\mathrm{INF}-\alpha$ in the plaque and no-plaque groups. (A) Serum CXCL16 levels. (B) Serum TNF- $\alpha$ levels. ${ }^{*} \mathrm{p}<0.05$ vs. no-plaque group; ${ }^{\text {ns }} \mathrm{p}<0.01$ vs. no-plaque group. CXCL16, CXC ligand 16; TNF- $\alpha$, tumor necrosis factor- $\alpha$.

\section{Discussion}

CAHD patients usually exhibit a variety of heart and nervous function alterations due to blood flow abnormalities. CADH greatly endangers the life and health of the middle-aged and elderly people in any population, which is why it is one of the most important diseases that need to be better studied. The pathological mechanism of CAHD is complex, involving
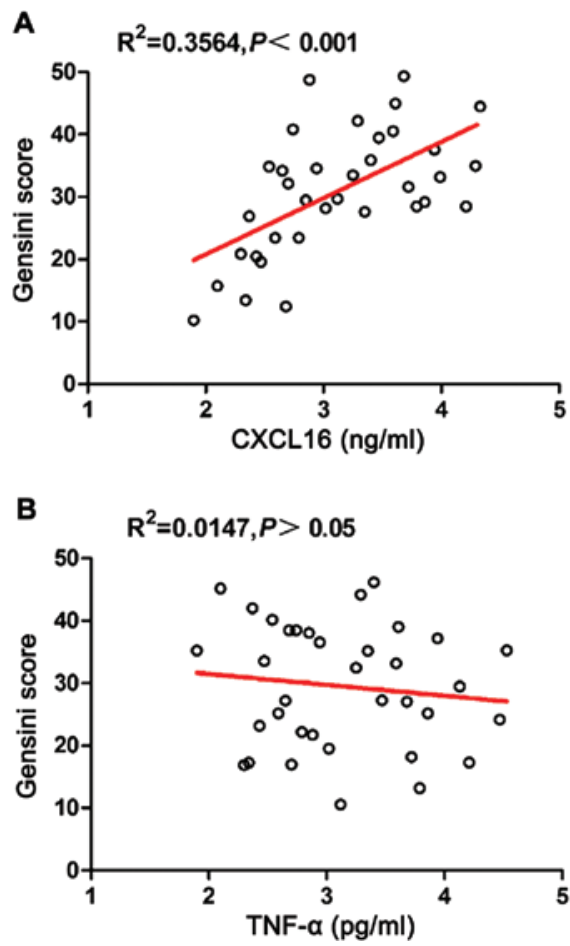

Figure 3. Correlation between serum levels of CXCL16 and TNF- $\alpha$ and Gensini scores. CXCL16, CXC ligand 16; TNF- $\alpha$, tumor necrosis factor- $\alpha$.

Table I. Correlations between serum levels of CXCL16 and TNF- $\alpha$ and clinical signs in the CAHD group.

\begin{tabular}{lccccc}
\hline & \multicolumn{2}{c}{ CXCL16 } & & \multicolumn{2}{c}{ TNF- $\alpha$} \\
\cline { 2 - 3 } \cline { 5 - 6 } Clinical index & r value & P-value & & r value & P-value \\
\hline $\begin{array}{l}\text { Systolic pressure } \\
\text { (mmHg) }\end{array}$ & 0.198 & 0.124 & -0.208 & 0.412 \\
$\begin{array}{l}\text { Diastolic pressure } \\
\text { (mmHg) }\end{array}$ & -0.168 & 0.156 & & 0.166 & 0.241 \\
$\begin{array}{l}\text { Fasting blood } \\
\text { glucose (mmol/l) }\end{array}$ & 0.067 & 0.561 & & -0.054 & 0.871 \\
$\begin{array}{l}\text { Heart rate } \\
\text { (bp/min) }\end{array}$ & 0.176 & 0.314 & & 0.114 & 0.142 \\
TC (mmol/l) & 0.196 & 0.177 & & 0.188 & 0.255 \\
$\begin{array}{l}\text { TG (mmol/l) } \\
\text { LDL-C (mmol/l) }\end{array}$ & -0.097 & 0.597 & $0.027^{\mathrm{a}}$ & -0.163 & 0.331 \\
HDL-C (mmol/l) & -0.179 & 0.274 & 0.075 & 0.752 \\
\hline
\end{tabular}

CXCL16, CXC ligand 16; TNF- $\alpha$, tumor necrosis factor- $\alpha$; CAHD, coronary atherosclerotic heart disease; TC, total cholesterol; TG, triglyceride; LDL-C, low-density lipoprotein cholesterol; HDL-C, high-density lipoprotein cholesterol. ${ }^{\mathrm{a}} \mathrm{p}<0.05$.

oxidative stress, immune inflammatory reactions, AS plaque formation and apoptosis. The occurrence of AS and instability of AS plaques are the basis of $\mathrm{CADH}$ pathogenesis. Vascular inflammatory reactions play an important role in AS plaque formation, which can promote coronary artery wall lesions thus leading to CAHD. 
Chemokines, such as the monocyte chemoattractant protein-1 and interleukin-8, activate and direct leukocytes to atherosclerotic lesions. CXCL16 is a recently found CXC family chemokine. It is expressed in soluble form across the cell membrane. CXCL16 has been closely related to the pathogenic formation of AS regulating inflammation and lipid metabolism (11). Its interaction with CXCR6 receptor on the surface of T lymphocytes directs the migration of activated Tlymphocytes to the AS lesion tissue. $\mathrm{T}$ lymphocytes can promote plaque formation and thromobosis vulnerability by locally secreting multiple cytokines and matrix metalloproteinases (MMPs) (12). In addition, CXCL16 combines with macrophages accelerating the endocytosis of oxidized LDL (Ox-LDL) to form foam cells (13), and it acts as an angiogenic factor inducing microvascular formation in AS plaques (14). Finally, CXCL16 activates CB8 ${ }^{+} \mathrm{T}$ cells, leading to apoptosis in the surrondings of an AS plaque (15). CXCL16 expression can be upregulated by IFN- $\gamma$ in AS plaques, suggesting that it enhances the role of the inflammatory response in AS lesions by means of a positive feedback loop mechanism (16). Thus, inflammation reactions affect the instability of AS plaques and markers of inflammation can be used to evaluate the risk of coronary heart disease. The levels of CXCL16 can reflect the upstream and downstream inflammation pathways, which can be used for the clinical prognosis and diagnosis of various cardiovascular and cerebrovascular diseases. Indeed, the soluble CXCL16 has been utilized as a biological marker of rheumatoid arthritis and systemic lupus erythematosus and other diseases (17).

TNF- $\alpha$ is a cytokine that can cause tumor cell necrosis. In recent years, TNF- $\alpha$ has been shown to be of great importance in the inflammation response (18). It can increase the release of soluble CXCL16 from endothelial cells and smooth muscle cells (SMCs) and it is abnormally highly expressed in patients with coronary heart disease (19). TNF- $\alpha$ is involved in the pathological process of AS by increasing inflammatory cells in the injured tissue, assisting vascular smooth muscle remodeling and exerting a negative inotropic effect on the myocardium, in which myocardial fiber necrosis and coronary artery ischemia can cause myocarditis and release of even more inflammatory mediators (20).

Our study found a significant increase in serum levels of CXCL16 and TNF- $\alpha$ in CAHD patients, indicating that they may be involved in the pathological process of CAHD. The levels of CXCL16 were significantly higher in the plaque group than that in the no-plaque group and had a significantly positive correlation with severity of CAHD. But, there were no significant differences in TNF- $\alpha$ levels between the two CADH groups and TNF- $\alpha$ was not significantly correlated with the degree of lesion present in $\mathrm{CADH}$ patients. Our findings suggest that CXCL16 can be used as a clinical diagnostic marker and illness evaluation factor in AS plaque formation and development of CAHD. Additionally, the TNF- $\alpha$ level was not significantly correlated with clinical variables studied, but CXCL16 had a significantly positive correlation with the plasma LDL-C levels in patients with CAHD, revealing that CXCL16 can be used as a valuable tool for diagnosis, treatment assessment and prognosis of CAHD.

In conclusion, in patients with CAHD, CXCL6 acts as an independent risk factor that can be used as a reliable biological marker for diagnosis, disease evaluation and clinical prognosis of $\mathrm{CADH}$.

\section{References}

1. McMillan RL: Coronary atherosclerotic heart disease. N C Med J 19: 147-149, 1958.

2. Hansson GK: Inflammation, atherosclerosis, and coronary artery disease. N Engl J Med 352: 1685-1695, 2005.

3. Kaperonis EA, Liapis CD, Kakisis JD, Dimitroulis D and Papavassiliou VG: Inflammation and atherosclerosis. Eur J Vasc Endovasc Surg 31: 386-393, 2006.

4. Stoll $\mathrm{G}$ and Bendszus M: Inflammation and atherosclerosis: Novel insights into plaque formation and destabilization. Stroke 37: 1923-1932, 2006.

5. Cannon JG: Inflammatory cytokines in nonpathological states. News Physiol Sci 15: 298-303, 2000.

6. Barlic J and Murphy PM: Chemokine regulation of atherosclerosis. J Leukoc Biol 82: 226-236, 2007.

7. Abel S, Hundhausen C, Mentlein R, Schulte A, Berkhout TA, Broadway N, Hartmann D, Sedlacek R, Dietrich S, Muetze B, et al: The transmembrane CXC-chemokine ligand 16 is induced by IFN-gamma and TNF-alpha and shed by the activity of the disintegrin-like metalloproteinase ADAM10. J Immunol 172: 6362-6372, 2004.

8. Shimaoka T, Kume N, Minami M, Hayashida K, Kataoka H, Kita T and Yonehara S: Molecular cloning of a novel scavenger receptor for oxidized low density lipoprotein, SR-PSOX, on macrophages. J Biol Chem 275: 40663-40666, 2000.

9. Schreyer SA, Vick CM and LeBoeuf RC: Loss of lymphotoxinalpha but not tumor necrosis factor-alpha reduces atherosclerosis in mice. J Biol Chem 277: 12364-12368, 2002.

10. Nowaczenko M, Sarzyńska-Długosz I and Członkowska A: Prevalence of carotid arteries atherosclerotic changes in ischemic stroke patients. Neurol Neurochir Pol 37: 27-36, 2003 (In Polish).

11. Smith C, Halvorsen B, Otterdal K, Waehre T, Yndestad A, Fevang B, Sandberg WJ, Breland UM, Frøland SS, Oie E, et al: High levels and inflammatory effects of soluble CXC ligand 16 (CXCL16) in coronary artery disease:down-regulatory effects of statins. Cardiovasc Res 79: 195-203, 2008.

12. Agostini C, Cabrelle A, Calabrese F, Bortoli M, Scquizzato E, Carraro S, Miorin M, Beghè B, Trentin L, Zambello R, et al: Role for CXCR6 and its ligand CXCL16 in the pathogenesis of T-cell alveolitis in sarcoidosis. Am J Respir Crit Care Med 172: 1290-1298, 2005.

13. Minami M, Kume N, Shimaoka T, Kataoka H, Hayashida K, Yonehara S and Kita T: Expression of scavenger receptor for phosphatidylserine and oxidized lipoprotein (SR-PSOX) in human atheroma. Ann N Y Acad Sci 947: 373-376, 2001.

14. Zhuge X, Murayama T, Arai H, Yamauchi R, Tanaka M, Shimaoka T, Yonehara S, Kume N, Yokode M and Kita T: CXCL16 is a novel angiogenic factor for human umbilical vein endothelial cells. Biochem Biophys Res Commun 331: 1295-1300, 2005.

15. Aslanian AM and Charo IF: Targeted disruption of the scavenger receptor and chemokine CXCL16 accelerates atherosclerosis. Circulation 114: 583-590, 2006.

16. Wuttge DM,Zhou X, Sheikine Y,Wågsäter D, Stemme V,Hedin U, Stemme S, Hansson GK and Sirsjö A: CXCL16/SR-PSOX is an interferon-gamma-regulated chemokine and scavenger receptor expressed in atherosclerotic lesions. Arterioscler Thromb Vasc Biol 24: 750-755, 2004.

17. Wu T, Xie C, Wang HW, Zhou XJ, Schwartz N, Calixto S, Mackay M, Aranow C, Putterman C and Mohan C: Elevated urinary VCAM-1, P-selectin, soluble TNF receptor-1, and CXC chemokine ligand 16 in multiple murine lupus strains and human lupus nephritis. J Immunol 179: 7166-7175, 2007.

18. Carswell EA, Old LJ, Kassel RL, Green S, Fiore N and Williamson B: An endotoxin-induced serum factor that causes necrosis of tumors. Proc Natl Acad Sci USA 72: 3666-3670, 1975.

19. Wang YN, Che SM and Ma AQ: Clinical significance of serum cytokines IL-1beta, sIL-2R, IL-6, TNF-alpha, and IFN- $\gamma$ in acute coronary syndrome. Chin Med Sci J 19: 120-124, 2004.

20. Trepels T, Zeiher AM and Fichtlscherer S: Acute coronary syndrome and inflammation. Biomarkers for diagnostics and risk stratification. Herz 29: 769-776, 2004 (In German).

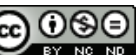

This work is licensed under a Creative Commons Attribution-NonCommercial-NoDerivatives 4.0 International (CC BY-NC-ND 4.0) License. 